\title{
Experimental Brain Research
}

\section{ELECTROPHYSIOLOGICAL EVIDENCE FOR A SELF PROCESSING ADVANTAGE DURING AUDIOVISUAL SPEECH INTEGRATION

\author{
--Manuscript Draft--
}

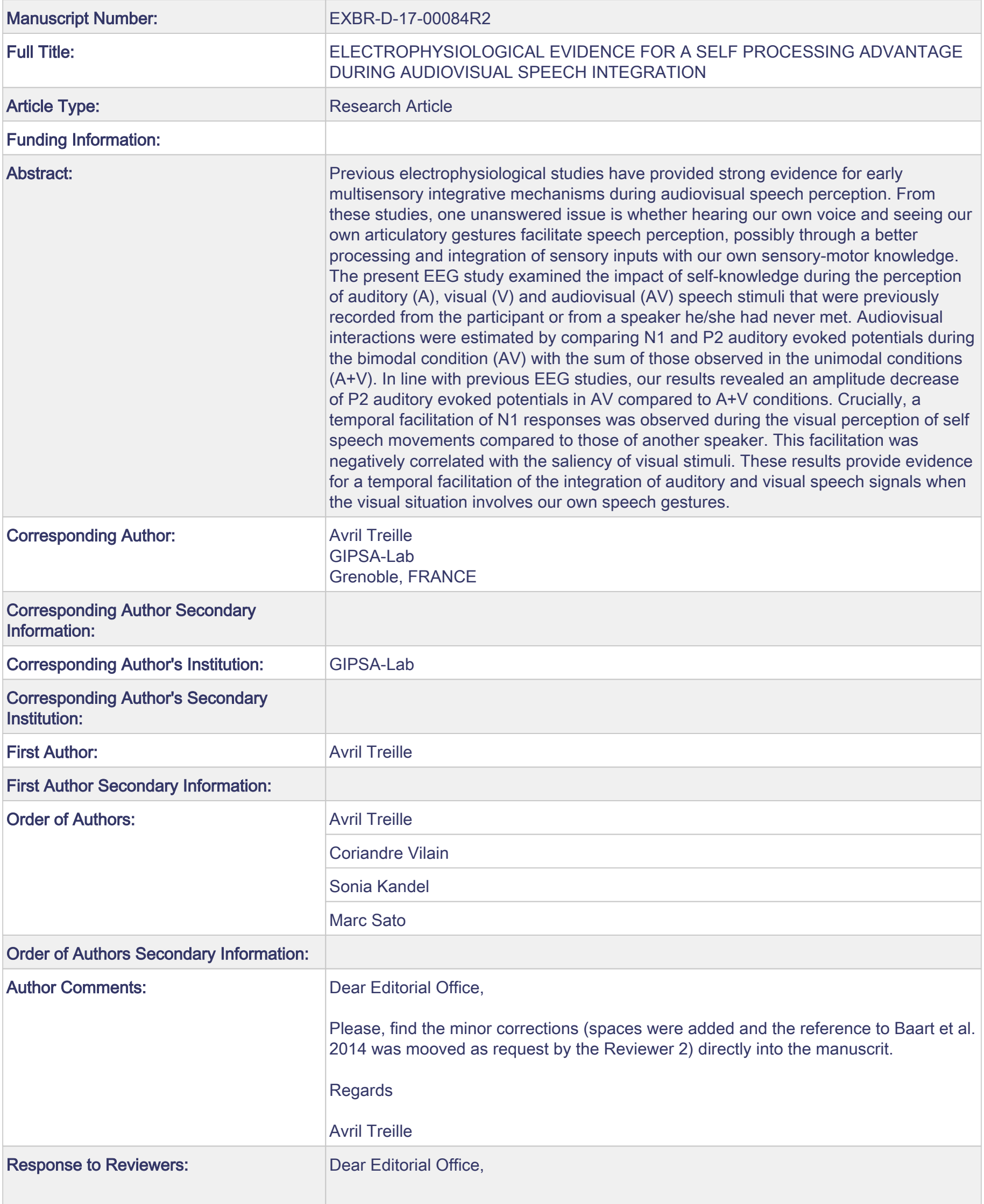


Please, find the minor corrections (spaces were added and the reference to Baart et al. 2014 was mooved as request by the Reviewer 2) directly into the manuscrit.

Regards

Avril Treille 


\section{ELECTROPHYSIOLOGICAL EVIDENCE FOR A SELF PROCESSING ADVANTAGE DURING AUDIOVISUAL SPEECH INTEGRATION}

Avril Treille ${ }^{1, C A}$, Coriandre Vilain ${ }^{1}$, Sonia Kandel ${ }^{1} \&$ Marc Sato $^{2}$

${ }^{1}$ GIPSA-lab, Département Parole \& Cognition, CNRS \& Grenoble Université, France

${ }^{2}$ Laboratoire Parole \& Langage, CNRS \& Aix-Marseille Université, France

${ }^{\mathrm{CA}}$ Corresponding author:

Avril Treille

Gipsa-lab, Université Stendhal

1180, avenue Centrale BP25

38031 GRENOBLE CEDEX 9

Email: avril.treille@gipsa-lab.grenoble-inp.fr

Phone: +33 (0)4 76824128 


\section{ABSTRACT}

Previous electrophysiological studies have provided strong evidence for early multisensory integrative mechanisms during audiovisual speech perception. From these studies, one unanswered issue is whether hearing our own voice and seeing our own articulatory gestures facilitate speech perception, possibly through a better processing and integration of sensory inputs with our own sensory-motor knowledge. The present EEG study examined the impact of self-knowledge during the perception of auditory (A), visual (V) and audiovisual (AV) speech stimuli that were previously recorded from the participant or from a speaker he/she had never met. Audiovisual interactions were estimated by comparing N1 and P2 auditory evoked potentials during the bimodal condition (AV) with the sum of those observed in the unimodal conditions $(A+V)$. In line with previous $E E G$ studies, our results revealed an amplitude decrease of $\mathrm{P} 2$ auditory evoked potentials in $\mathrm{AV}$ compared to $\mathrm{A}+\mathrm{V}$ conditions. Crucially, a temporal facilitation of $\mathrm{N} 1$ responses was observed during the visual perception of self speech movements compared to those of another speaker. This facilitation was negatively correlated with the saliency of visual stimuli. These results provide evidence for a temporal facilitation of the integration of auditory and visual speech signals when the visual situation involves our own speech gestures.

Keywords: Self recognition, speech perception, audiovisual integration, EEG. 


\section{INTRODUCTION}

Lip-reading alone is not enough to understand an utterance. However, information from the speaker's face (e.g., lip movements) is known to improves auditory speech perception. Several studies indicate that visual speech information enhances auditory speech intelligibility in noisy environments (Sumby \& Pollack, 1954; Benoît, Mohamadi \& Kandel, 1994), facilitates phoneme identification of nonnative phonemes (Navarra \&Soto-Faraco, 2005; Burfin et al., 2014) or even contributes to the comprehension of complex content (Reisberg, McLean \& Goldfield, 1987). In addition, in laboratory experimental situations, visual incongruent information (/ga/) when added to an auditory syllable (/ba/) can generate a new percept $(/ \mathrm{da} /)$ different from both the auditory and visual syllables. This perceptual illusion was first displayed by McGurk and MacDonald in 1976 and strikingly underlines the complementarity and intimate interaction between auditory and visual speech information. Interestingly, visual information is not the only way to facilitate auditory speech decoding. Behavioral studies on tactile and audio-tactile speech perception also demonstrate that perceiving orofacial gestures of the speaker through the hand (via the TADOMA method; see Alcorn, 1932)can facilitate syllable discrimination (Reed et al., 1985, 1992; Reed et al., 1982; Fowler \& Dekle, 1991; Gick et al., 2008; Sato et al., 2010; Treille et al., 2014a, 2014b).

At the brain level, electro-encephalographic (EEG) and magneto-encephalographic (MEG) studies demonstrate that N1/M1 and P2 auditory evoked potentials are attenuated and speeded up when an auditory syllable is combined with visual or tactile information from the speaker's face (Klucharev et al., 2003; Besle et al., 2004; van Wassenhove et al., 2005; Stekelenburg \& Vroomen, 2007; Arnal et al., 2009; Pilling, 2010; Vroomen \& Stekelenburg, 2010; Frtusova, Winneke \& Phillips, 2013; Kaganovich \& Schumaker, 2014; Treille et al., 2014a, 2014b; Baart et al., 2014; Baart \& Samuel, 2015). This temporal facilitation of latency (onset of neural processing) and amplitude suppression (size of neural population and activation synchrony during the component generation) of N1/M1 and P2 auditory evoked potentials is thought to reflect early multisensory integrative mechanisms through visual predictions of the incoming auditory events. However, the speech specific nature of these effects remains controversial. Indeed, Stekelenburg and Vroomen (2007) and Vroomen and Stekelenburg (2010) observed similar N1 latency and amplitude decreases during the observation of biological transitive (spoon hitting a cup, handclapping) and intransitive (Tearing of paper) non-speech actions, and even during the observation of non-biological actions (a pure tone synchronized with a deformation of a rectangle, or a collision of moving disks).These studies suggested that N1 and P2modulations would reflect different aspects of audiovisual integration mechanisms (van Wassenhove et al., 2005; Arnal et al., 2009; Baart et al., 2014). There would be a non speech-specific stage in audiovisual integration that processes the early arrival of visual information. This would be reflected by N1 latency and amplitude 
modulations. A subsequent speech-specific featural phonetic stage would be reflected in P2 modulations (see Baart et al., 2014 for a review).

Neuroimaging studies further demonstrate the existence of specific brain areas playing a key role in the audiovisual integration of speech. In particular, audiovisual speech perception has an impact on the activity of unisensory visual and auditory regions (the visual motion-sensitive cortex, V5/MT, and the Heschl's gyrus) as well as multisensory regions (the posterior part of the left superior temporal gyrus/sulcus, pSTS/pSTG), when compared to auditory and visual unimodal conditions (Calvert, Campbell and Brammer, 2000; Callan et al., 2003, 2004; Skipper et al., 2005, 2007). Interestingly, the premotor cortex-that is involved in speech production and is part of the dorsal stream (Hickok \& Poeppel, 2007) might also play a role in audiovisual speech integration mechanisms. Indeed, previous studies on audiovisual speech perception demonstrated stronger activation of this premotor region during the presentation of bimodal speech stimuli compared to auditory and visual only conditions (Campbell et al., 2001; Calvert \& Campbell, 2003; Watkins, Strafella \& Paus, 2003; Watkins \& Paus, 2004; Skipper et al., 2005, 2007; Sato et al., 2010). This occurred during the presentation of incongruent stimuli compared to congruent ones (Jones \&Callan, 2003; Ojanen et al., 2005; Pekkola et al., 2006) and also in the case of degraded visual or auditory speech signals (Callan et al., 2003, 2004). Taken together - and although the debate is still open- these studies, support the idea that motor knowledge used to produce speech sounds might constrain phonetic decoding of the sensory inputs. This comforts, to a certain extent, the motor and sensorimotor theories of speech perception and language comprehension (Liberman \& Mattingly, 1985; Skipper et al., 2007; Schwartz et al., 2012; Pickering \& Garrod, 2013) and supports the long-standing proposal that perception and action are two closely linked processes.

From these studies on audiovisual speech perception, one intriguing question is whether hearing our own voice and seeing our own articulatory gestures facilitate speech perception, possibly through a better processing and integration of sensory inputs with our own sensory-motor knowledge. From this question, a few behavioral studies have provided contrasted results. Tye-Murray and colleagues $(2013,2014)$ demonstrated that, during sentence lip-reading, participants recognize better their visual productions than those of others. In contrast, Aruffo and Shore (2012) found a self-auditory but not a self-visual advantage during the presentation of incongruent audiovisual speech stimuli. Other behavioral studies attempted to show a self-processing effect during audiovisual syllable perception, but the results were not concluding (Schwartz and Savariaux, 2001).

The present study examined whether self-information processing constitutes an advantage during audiovisual speech integration. We used EEG to examine N1 and P2 auditory evoked potentials during 
the perception of auditory and/or visual speech stimuli that were previously recorded from the participant (self) and a speaker he/she had never met (other). For each participant, eight conditions were tested, consisting on four distinct modalities: an auditory modality $\left(A_{\text {self }}, A_{\text {other }}\right)$, a visual modality $\left(\mathrm{V}_{\text {self }}, \mathrm{V}_{\text {other }}\right)$, an audiovisual modality $\left(\mathrm{A}_{\text {self }} \mathrm{V}_{\text {self }}, \mathrm{A}_{\text {other }} \mathrm{V}_{\text {other }}\right)$ and an audiovisual modality with incongruent speakers in which the acoustic and visual signals were produced by the participant and the other speaker respectively $\left(A_{\text {self }} V_{\text {other }}, A_{\text {other }} V_{\text {self }}\right)$. The audiovisual modality with incongruent speakers was designed to determine whether a possible self-effect comes from auditory or visual information. Using an additive model, we tested whether N1/P2 auditory evoked potentials were attenuated and speeded up during audiovisual conditions compared to the sum of those observed in unimodal conditions, and whether these effects were modulated by a self-processing advantage. 


\section{METHOD}

\section{PARTICIPANTS}

Eighteen healthy adults participated in the study (12 females; mean age $23, S D \pm 5$ years). All the participants were right-handed native French speakers, had normal or corrected-to-normal vision and reported no history of speaking, hearing or motor disorders. They gave written consent for their participation in the study. They were compensated for the time spent in the study. The study received approval by the Grenoble Alpes University Ethical Committee (CERNI, N²013-12-24-33).

\section{STIMULI}

Recording - We recorded 10 utterances of /apa/, /ata/ and /aka/ sequences of each participant in a soundproof room. Previous research on audiovisual speech perception has shown that these sequences correspond to a gradient of visuo-labial saliency: the unvoiced bilabial / $p /$ stop consonant is more salient visually than unvoiced alveolar stop consonant $/ \mathrm{t} /$ and in turn stop consonant velar $/ \mathrm{k} /$ unvoiced (e.g., van Wassenhove et al., 2005 for an EEG study). Moreover, these stop consonants have precise acoustics onsets, which is crucial for the EEG analyses we intended to carry out (see below). Then, we selected four utterances of each sequence for each participant on the basis of visual and acoustical durations (using Adobe Premiere, Adobe Systems, and Praat software; Boersma \& Weenink, 2013).

Stimulus preparation-The movies were created on the basis of 30 frames (1200 ms) before the acoustic burst and 5 frames $(200 \mathrm{~ms}$ ) after it, for a total duration of $1400 \mathrm{~ms}$ for all the stimuli. Prior to generating movies, we extracted the acoustic signal and erased the first vowel /a/ so that all the audio signals began with a $1200 \mathrm{~ms}$ silence. This procedure allows building stimuli with the same duration of an initial neutral mid-open mouth position of each participant (for examples on AV stimuli see supplementary material). Then, we merged the audio and video signals in four different types of movies:

-Auditory modality (A): the movie consisted of a fixed image of the last frame before the acoustic onset during the initial vowel /a/ dubbed on the acoustic signal.

-Visual modality (V): The movie consisted of the visual input without the sound.

-Audiovisual modality (AV): The movie consisted of both the auditory and visual signals.

-Audiovisual modality with incongruent speakers (AV incongruent speakers): The movie consisted of the acoustic signal of the speaker dubbed with the visual signal of the same syllable but produced by another participant (see below for the matching method).

Participant pair matching - Because of possible idiosyncrasy or production differences between participants that might cause facilitation or perturbation of visual or auditory stimuli recognition, each 
participant was associated to an unknown participant (same gender and equivalent age). Each pair of participants was therefore presented with the same set of stimuli from both participants. With this procedure, a possible self effect cannot therefore be attributed to possible idiosyncrasy differences.

Our experiment therefore consisted of 9 pairs of participants. To each participant we presented both her/his own productions and those of her/his unknown partner (see Figure 1).For each participant, eight conditions were tested, consisting on four distinct modalities applied either on the participant her/himself (self) or the unknown speaker(other): an auditory modality $\left(A_{\text {self }}, A_{\text {other }}\right.$ ), a visual modality $\left(\mathrm{V}_{\text {self, }}, \mathrm{V}_{\text {other }}\right)$, an audiovisual modality $\left(\mathrm{A}_{\text {self }} \mathrm{V}_{\text {self, }}, \mathrm{A}_{\text {other }} \mathrm{V}_{\text {other }}\right)$ and an audiovisual modality with incongruent speakers in which the acoustic and visual signals were produced by the participant and the other speaker $\left(A_{\text {self }} V_{\text {other, }}, A_{\text {other }} V_{\text {self }}\right)$. The audiovisual modality with incongruent speakers was designed to determine whether a possible self-effect comes from auditory or visual information. With this procedure, a total of 864 stimuli were created (18 speakers $\times 4$ modalities $\times 3$ syllables $\times 4$ utterances).
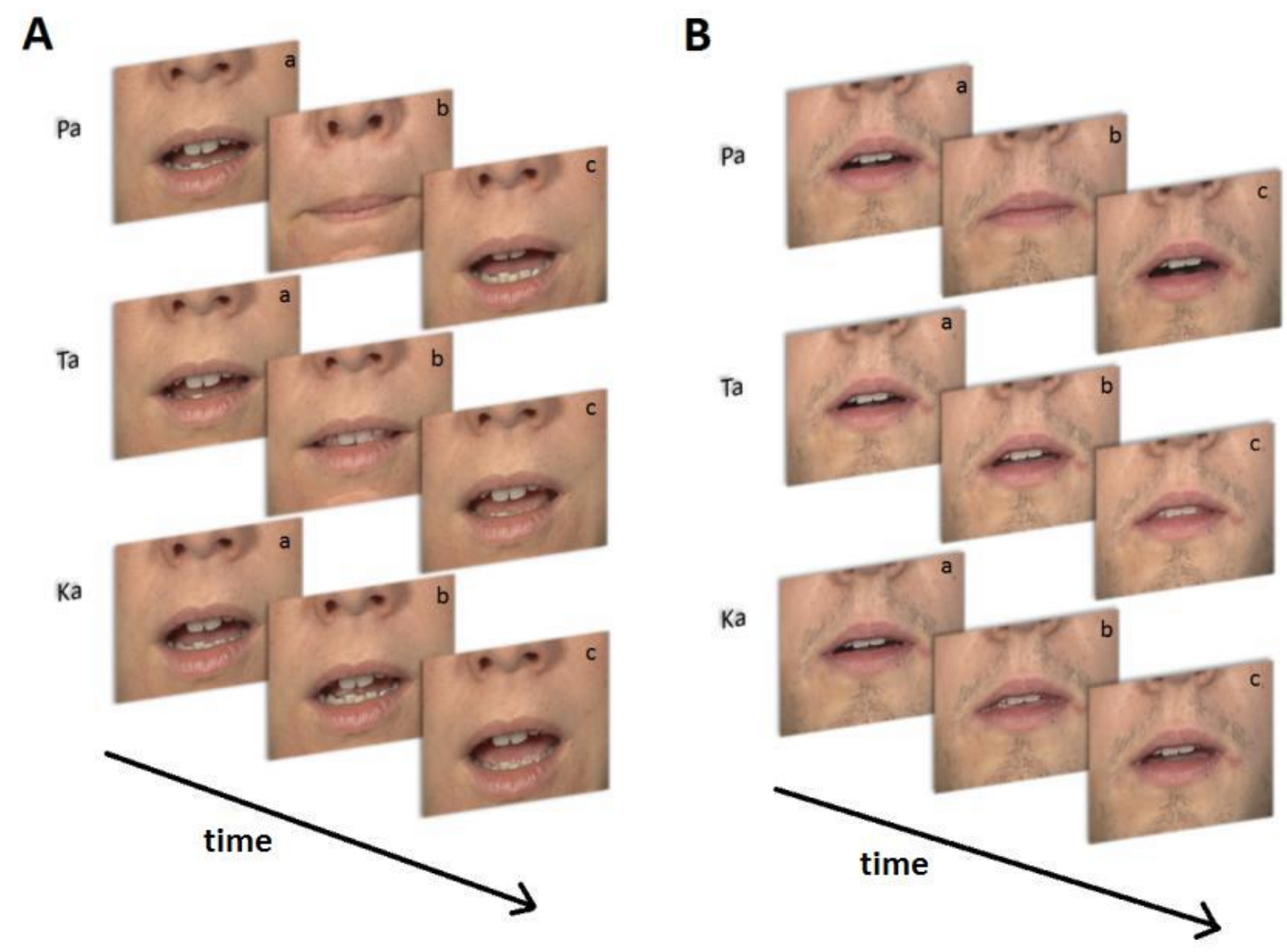

Figure 1: Examples of the visual stimuli for two participants $(A, B)$. Each utterance begins with the mouth open (a); is followed by the stop consonant (b); and ends with the second /a/ vowel (c). 


\section{EXPERIMENTAL PROCEDURE}

The participants sat in front of a computer monitor at a distance of approximately $50 \mathrm{~cm}$. The acoustic stimuli were presented at a comfortable sound level through loudspeakers, with the same sound level set for all participants (frame-rate of the video recordings: 25 images/sec, refresh-rate of the monitor: $60 \mathrm{~Hz}$ ). The software Presentation (Neurobehavioral Systems, Albany, CA) controlled stimulus presentation and recorded the participants' responses. The participants were instructed to identify the syllable presented by the movies by pressing a key on the keyboard with their left hand. It was a three-alternative $/ \mathrm{pa} / \mathrm{l} / \mathrm{ta} /$ and $/ \mathrm{ka} /$ forced-choice identification task. In order to dissociate sensory/perceptual responses from motor responses on EEG data, a brief single audio beep was delivered $600 \mathrm{~ms}$ after the end of each stimulus. The participants had to respond after this audio beep. The experiment consisted of 576 trials presented in a pseudo-randomized sequence, with 24 trials in each condition (4 modalities ( $A, V, A V, A V$ with incongruent speakers) $\times 2$ speakers (self and other) $\times 3$ syllables (/pa/, / ta/ and $/ \mathrm{ka} /) \times 24$ trials). The inter-trial interval was set at $3 \mathrm{~s}$ and the response key designation was fully counterbalanced across participants. 
A-self A-other AV-self AV-other AVi-self AVi-other V-self V-other

Oms
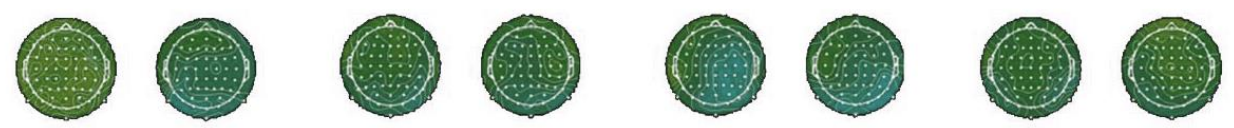

$50 \mathrm{~ms}$
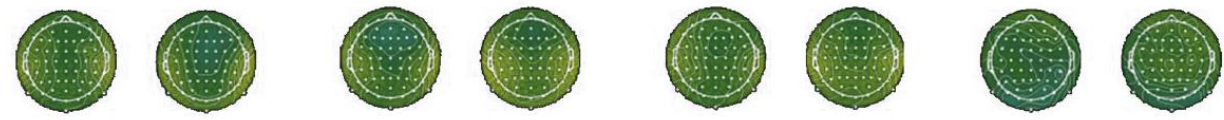

$100 \mathrm{~ms}$

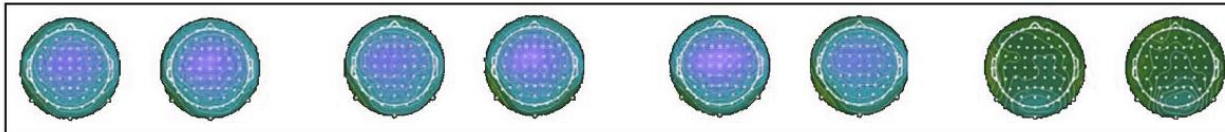

$150 \mathrm{~ms}$
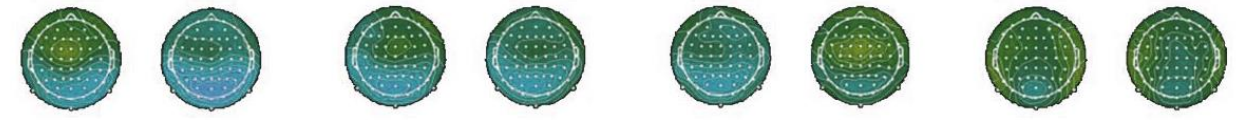

3uv

200ms
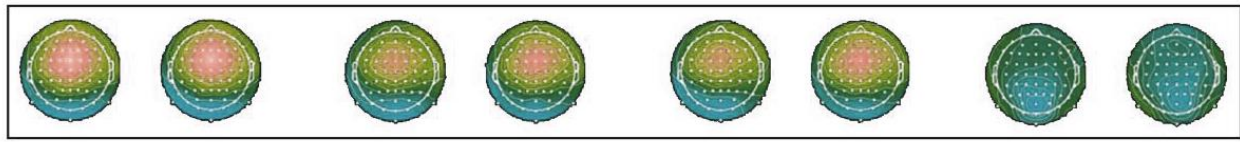

250ms
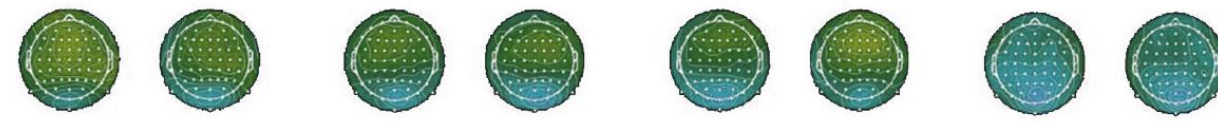

-3uv

$300 \mathrm{~ms}$
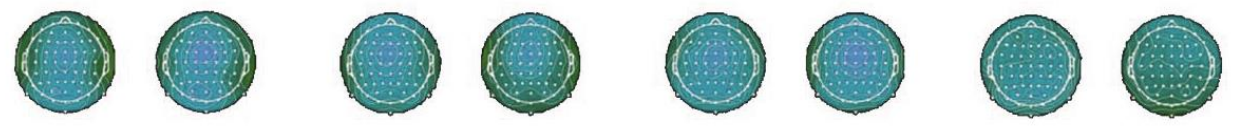

$350 \mathrm{~ms}$
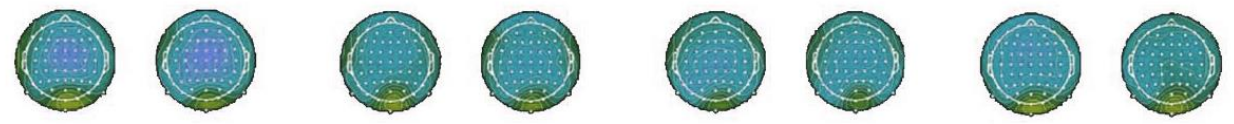

Figure 2: Topographic analysis conducted on all the participants and electrodes demonstrating a maximal response of N1/P2 auditory evoked potentials on fronto-central electrodes.

EEG data were recorded continuously from 64 scalp electrodes (Electro-Cap International, INC, according to the international 10-20 system) using the Biosemi Active Two AD-box EEG system operating at a $256 \mathrm{~Hz}$ sampling rate. Two additional electrodes served as reference (Common Mode Sens [CMS] active electrode) and ground (Driven Right Leg [DRL] passive electrode). One other external reference electrode was set at the top of the nose. Horizontal (HEOG) and vertical (VEOG) eye movements were recorded using an electro-oculogram with electrodes positioned the outer canthus of each eye as well as above and below the right eye. Before the experiment, the impedance of all electrodes was adjusted to get low offset voltages and stable DC.

EEG data were processed using the EEGLAB toolbox (Delorme and Makeig, 2004) running on Matlab (Mathworks, Natick, MA, USA). Since N1/P2 auditory evoked potentials have maximal response over central sites on the scalp (Scherg and Von Cramon, 1986; Näätänen and Picton, 1987), EEG data preprocessing and analyses were conducted on 6 representative fronto-central electrodes (F3, Fz, F4, $\mathrm{C} 3, \mathrm{Cz}, \mathrm{C} 4)$. This is in line with previous EEG studies on audiovisual speech perception and auditory 
evoked potentials (e.g. Pilling, 2010; Stekelenburg and Vroomen, 2007; van Wassenhove et al., 2005; electrodes demonstrated a maximal response of N1/P2 auditory evoked potentials on fronto-central electrodes (see Figure 2). This confirmed the reliability of our selection of fronto-central electrodes. EEG data were first off-line re-referenced to the nose recording and band-pass filtered using a twoway least-square FIR filtering $(1-20 \mathrm{~Hz})$. Data were then segmented into $1000 \mathrm{~ms}$ epochs including a $100 \mathrm{~ms}$ pre-stimulus baseline (from $-500 \mathrm{~ms}$ to $-400 \mathrm{~ms}$ relative to the acoustic syllable onset). Epochs with an amplitude change exceeding $\pm 60 \mathrm{uV}$ at any channel (including HEOG and VEOG channels) were rejected (on average, less than $6 \%$ ). For each participant and condition $\left(A_{\text {self }}, A_{\text {other, }}, V_{\text {self, }}, V_{\text {other, }}, A_{\text {self }} V_{\text {self, }}\right.$ $\left.A_{\text {other }} V_{\text {other }}, A_{\text {self }} V_{\text {other, }}, A_{\text {other }} V_{\text {self }}\right)$, the data were averaged on the 6 electrodes. Then the maximal amplitude and peak latency of auditory N1 and P2 evoked responses were determined on the EEG waveform using a fixed window (N1: 70-150 ms; P2: 150-250 ms).

\section{DATA ANALYSES}

\section{Behavioral analyses}

The percentage of correct responses was determined for each participant, syllable and modality. We conducted a three-way repeated-measures ANOVAs with speaker type (self vs. other), modality (A, V , AV, AV with incongruent speakers) and the syllable (/pa/, /ta/ and / ka/) as within-participants variables.

\section{EEG analyses}

Audiovisual integration - To test audiovisual speech integration, we used an additive model, with EEG responses in the bimodal conditions (AV) compared to the sum of auditory and visual EEG responses $(A+V)$. We conducted three-way repeated-measures ANOVAs on N1/P2 amplitudes and latencies with signal type (bimodal vs. sum), auditory speaker (self vs. other) and visual speaker (self, other or none) as within-participants factors.

Correlation between accuracy and EEG signals-To test the relation between the perceptual visual saliency and degree of integration observed on the EEG signals, we conducted Pearson correlation analyses. The analyses concerned the relation between visual accuracy and the modulations of either N1/P2 amplitude or latency. They were related to the difference between the bimodal conditions and the sum of unimodal conditions (e.g., EEG responses on $\left[A_{\text {self }} V_{\text {self }}-\left(A_{\text {self }}+V_{\text {self }}\right)\right]$ and $\left[A_{\text {other }} V_{\text {self }}-\left(A_{\text {other }}+\right.\right.$ $\left.\left.V_{\text {self }}\right)\right]$ correlated with $V_{\text {self }}$ Scores, or EEG responses on $\left[A_{\text {self }} V_{\text {other }}-\left(A_{\text {self }}+V_{\text {other }}\right)\right]$ and $\left[A_{\text {other }} V_{\text {other }}-\left(A_{\text {other }}\right.\right.$ $\left.\left.+V_{\text {other }}\right)\right]$ correlated with $V_{\text {other }}$ scores). 


\section{RESULTS}

\section{ACCURACY}

Overall, the mean proportion of correct responses was $94 \%$ (see Figure 3). The analyses revealed a main effect of presentation modality $(F(3,51)=67.6 ; p<.0001)$. The percentages of correct responses for the visual stimuli (83\%) were lower than for auditory (A: 98\%) and audiovisual stimuli (AV: 99\%; AVi: $98 \%)$. In addition, consonant saliency also yielded a main effect $(F(2,34)=23.3 ; p<.0001)$. The /pa/syllables were identified better (98\%) than the /ta/ (92\%) and in turn /ka/ (93\%) ones. Finally, the interaction between the presentation modality and the syllable was reliable $(F(6,102)=24.1 ; p<.0001)$. There was an effect of syllable saliency in the visual modality (V-/pa/: 99\%; V-/ta/: 75\%; V-/ka/: 74\%).

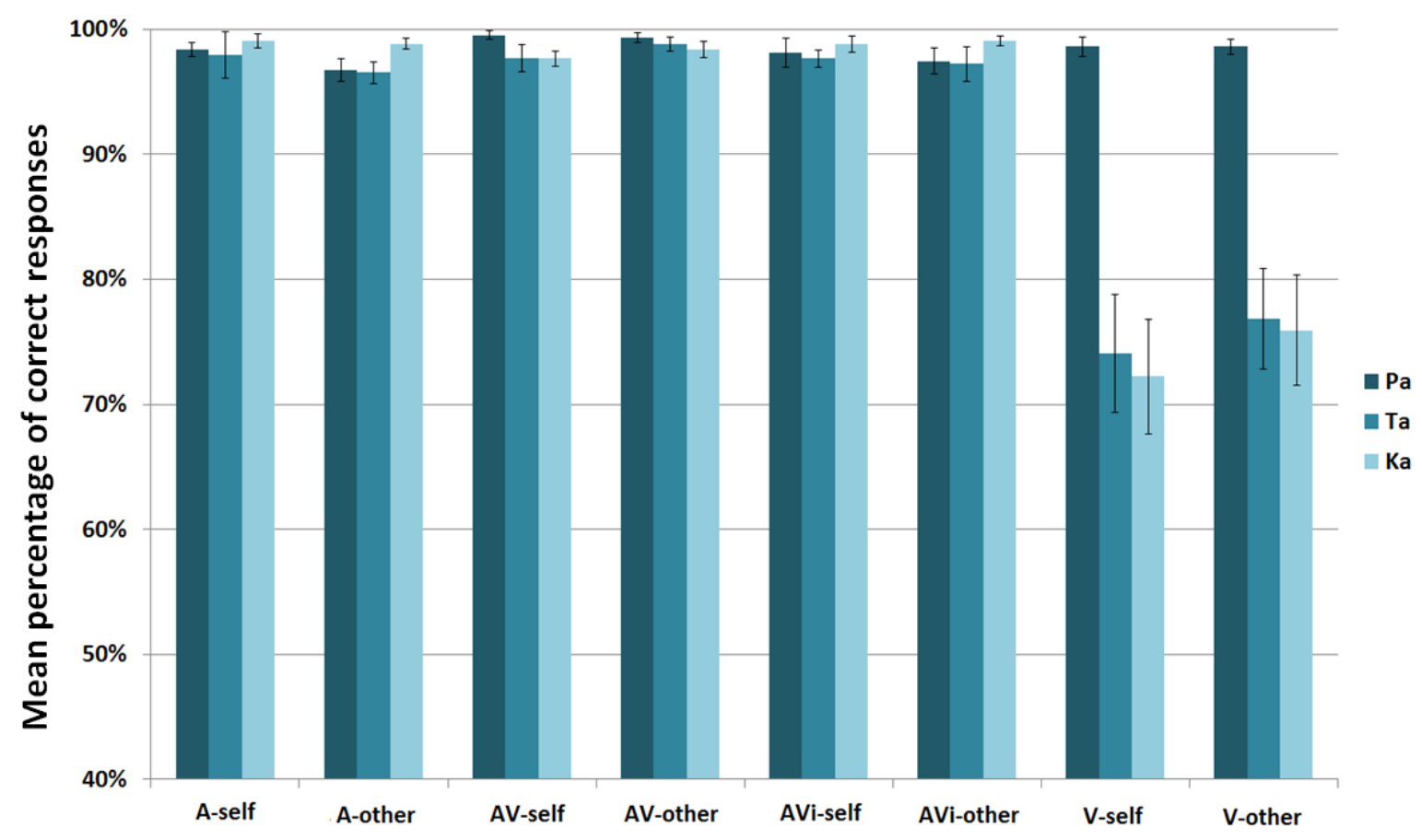

Figure 3: Mean percentage of correct responses observed for each speaker type, presentation modality and each syllable.

\section{EEG RESULTS}

Amplitude - None of the effects reached significance for N1 amplitude. There was a main effect of signal type for $\mathrm{P} 2$ amplitude $(F(1,16)=6.9 ; \mathrm{p}<.02$; see Figure 4). The amplitude was smaller for the bimodal conditions $(3.8 \mu \mathrm{V})$ than the sum of the auditory and visual signals $(4.7 \mu \mathrm{V})$.

Latency - Regarding the analyses on N1 latency, there was a significant effect of the visual speaker $(F(1,16)=8.2 ; p<.02$; see Figure 4$)$. There was a temporal facilitation during the perception of visual-self speech movements (107 ms) compared to visual-other speech movements (113 ms). No significant effects were found for P2 latency. 


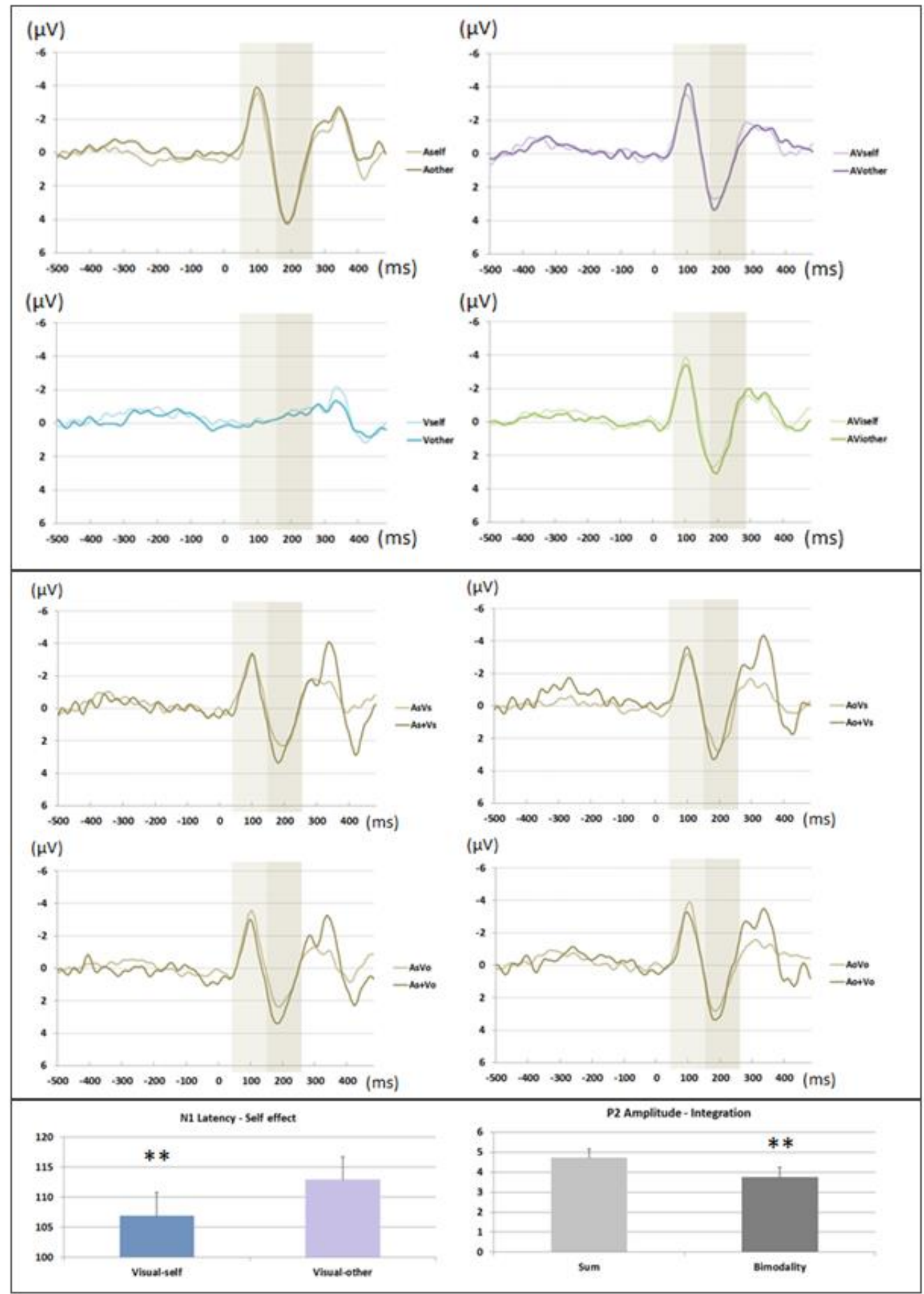

Figure 4: Top: Average event-related potentials on fronto-central electrodes related to each modality (A, AV, V, AVi) for "self" (light colour) and "other" (dark colour) conditions. Middle: Averaged event-related potentials on fronto-central electrodes related to the audiovisual conditions (AV) and the sum of unimodal conditions $(A+V)$ according to the auditory and the visual speakers (s: self; o: other). Bottom: Significant effects on N1 and P2. Left: Latency N1 decrease observed in audiovisual conditions for self compared to other visual movements. Right: Amplitude P2 decrease observed for AV compared to A+V. 


\section{CORRELATION BETWEEN BEHAVIORAL SCORES AND EEG SIGNALS}

Because a significant reduction of $\mathrm{N} 1$ latency was observed for self stimuli, we conducted additional correlation analyses between visual recognition scores and bot amplitude and latency of N1 and P2 PERs in order to test a possible relationship between the perceptual visual saliency and degree of integration observed on the EEG signals.

Amplitude - No significant correlation was found between EEG signals related to AV integration and the visual saliency of syllables for both $\mathrm{N} 1$ and P2 amplitude (N1: self: $r=.09 ; p<.63$; other: $r=.24$; $p<.16$; P2: self: $r=.22 ; p<.22$; other: $r=.18 ; p<.30$; see Figure 5$)$.

Latency - N1 latency difference between AV and A+V EEG responses related to the visual-self syllables was negatively correlated with the visual recognition scores ( $V$-self: $r=.41 ; p<.02$ ). No significant correlation was observed for the visual syllables from an unknown speaker ( $V$-other: $r=.01$; $\mathrm{p}<.94)$. Finally, no significant correlation was observed between P2 latency data related to the degree of integration of self and other visual information and visual accuracy (V-self: $r=.11 ; F(1,32)=0.32$; $p<.54 ;$ V-other: $r=.29 ; F(1,32)=2.95 ; p<.10$; see Figure 5$)$.
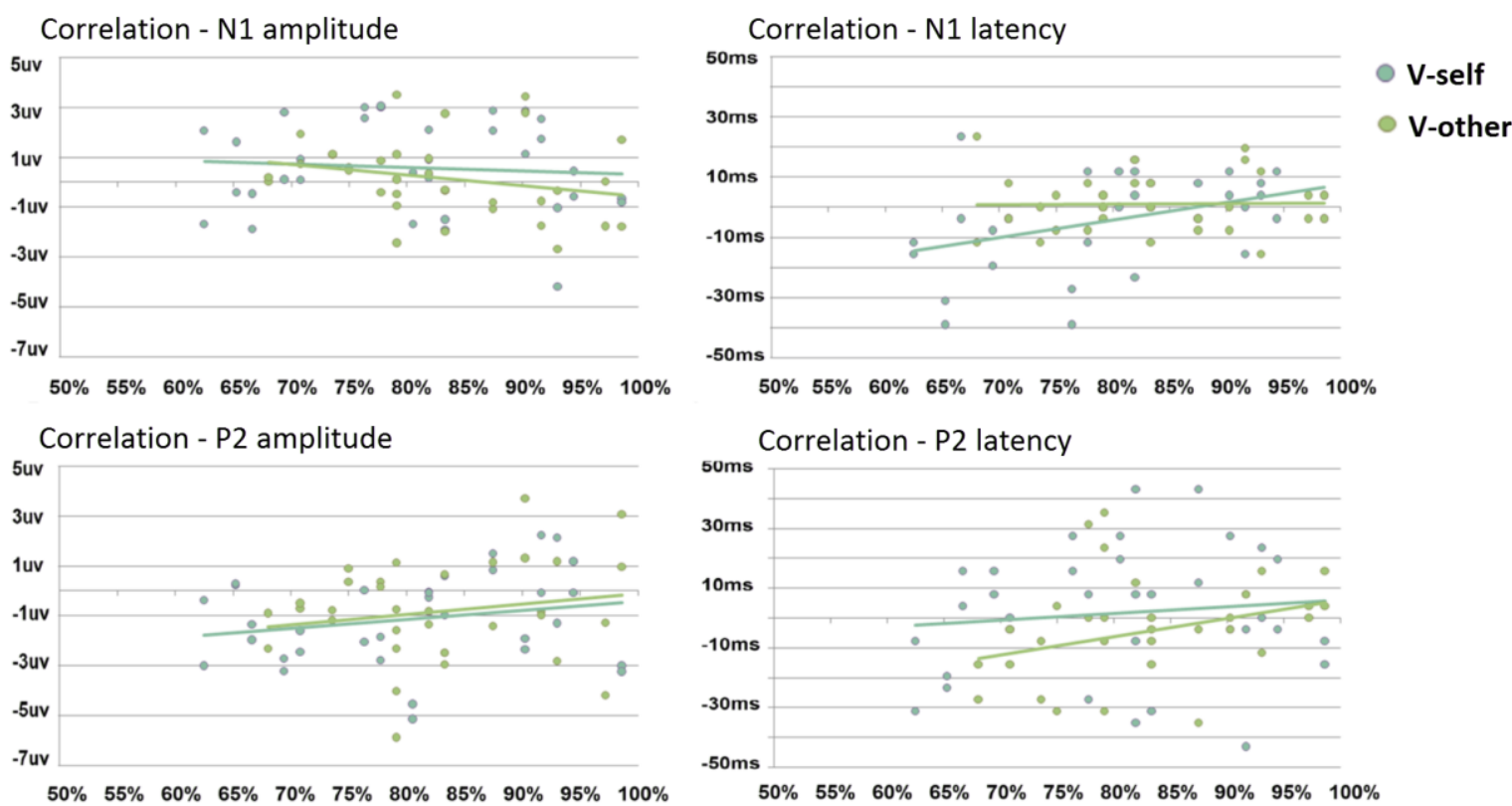

Figure 5: Correlation between the visual recognition scores for self and other visual movements ( $x$-axis) and the difference in amplitude and latency of $\mathrm{N} 1$ and $\mathrm{P} 2$ auditory evoked potentials between AV and A+V (yaxis). 


\section{DISCUSSION}

The present EEG study investigated a possible self-processing advantage during speech perception, and its related impact on audiovisual integration mechanisms. Two main results were observed. First and in line with previous EEG studies on audiovisual speech integration, we observed an amplitude decrease on P2 auditory evoked potentials during the bimodal presentation compared to the sum of auditory and visual unimodal responses. Crucially, during audiovisual speech integration, a temporal facilitation related to self lip movements was observed on N1 auditory evoked potentials, a facilitation that appears negatively correlated with the saliency of visual stimuli.

Previous studies on audiovisual speech integration demonstrated that bimodal presentations produce a decrease in N1 and/or P2 latency and amplitudes (Besle et al., 2004; van Wassenhove et al., 2005; Stekelenburg and Vroomen, 2007; Pilling, 2010; Baart et al., 2014; Treille et al., 2014a, 2014b) and latency (van Wassenhove et al., 2005; Stekelenburg et Vroomen, 2007; Baart et al., 2014; Treille et al., 2014a; see also Arnal et al., 2009 for similar results with MEG) when compared to auditory responses or to the sum of auditory and visual responses. These modulations of the N1/P2 responses are thought to reflect specific stages of audiovisual speech integration. N1 latency and amplitude modulations would reflect a non speech-specific stage while P2 latency shifts or amplitude decreases would rather be speech-specific and related to a featural phonetic stage (Baart et al., 2014). Using an additive model, our results revealed a P2 amplitude decrease during the bimodal presentation compared to the sum of the unimodal auditory and visual conditions. In line with previous studies (van Wassenhove et al., 2005; Stekelenburg and Vroomen, 2007; Pilling, 2010; Baart et al., 2014; Treille et al., 2014b), this result suggests that visual speech information affects ongoing auditory activity and further demonstrates the integration of auditory and visual speech signals. However, there were no differences on P2 latency, nor on N1 amplitude and latency. This contrasts with previous studies reporting latency shifts of auditory evoked responses and/or N1 amplitude decreases in the bimodal condition. Some aspects of the present experimental procedure might explain these differences. A first important point is related to the stimulus variability. In our experiment we presented four tokens of three syllables produced by two speakers. The above mentioned studies only presented one token of each presented syllable (van Wassenhove et al., 2005; Stekelenburg and Vroomen, 2007; Arnal et al., 2009; Baart et al., 2014) and/or a more limited number of syllables (i.e., one or two; Stekelenburg and Vroomen, 2007; Pilling, 2010; Vroomen and Stekelenburg, 2010; Baart et al., 2014; Treille et al., 2014a). In the present EEG experiment, the higher stimulus variability might have decreased eventual habituation/learning effects. This might have limited latency shifts on auditory evoked potentials. From that view, a recent meta-analysis suggests that variability across EEG/MEG studies on audiovisual speech integration may potentially be driven by many experimental, procedural, and methodological 
differences, such as the number and quality of stimuli, the sound intensity, the inter-trial interval, the

It is noteworthy that our behavioral results did not reveal any visual, auditory or audiovisual selfprocessing advantage. This contrasts with a behavioral study conducted by Tye-Murray and colleagues (2013). They showed that we lip-read more accurately sentences produced by ourselves than by other speakers. For the authors, these results provide support to the common coding theory (Prinz, 1997; Hommel et al., 2001), which posits that producing and perceiving share the same representations of motor plans. Because of this perceptuo-motor coupling, observing one's own action activates these motor plans to a greater extent than observing someone else's action. A reason for this divergence could reside on stimulus length. In the present study we used syllables whereas Tye-Murray et al used sentences. The use of short CV syllables therefore limited the quantity of visual information and facilitated correct responses (mean 94\%). Our results appear consistent however with the study by Aruffo and colleagues (2012) who did not find any visual self-processing advantage with participants presented with incongruent audiovisual syllables (McGurk stimuli), although self-voice appeared to weaken the illusion effect.

The major contribution of our EEG study is that it provides evidence for a visual self-processing advantage on N1 latency during audiovisual speech perception. More specifically, a temporal facilitation of audiovisual speech processing was observed when participants watched their own productions compared to those of another speaker. This facilitation was negatively correlated with the recognition score of visual self-stimuli. This suggests that the visual self-processing effect is linked to specific visual speech features of the presented syllables, like the place of articulation of the consonants (with their acoustic bursts here used as onsets for EEG analyses). Interestingly, this effect seems to be largely driven by visually "ambiguous" syllables, i.e. syllables that were the most difficult to identify (see Figure 5). Although this correlational result precludes any causal inferences, a plausible explanation could be that the difficulty to decode our own speech gestures would increase the degree of audiovisual integration and temporally facilitate auditory process.

In conclusion, the present EEG study provides the first electrophysiological evidence for a selfprocessing advantage during audiovisual speech integration. The observed temporal facilitation of N1 responses during the visual perception of self speech movements compared to those of another speaker suggest that perceiving our own articulatory gestures speed up auditory speech perception, possibly through a better processing and integration of sensory inputs with our own sensory-motor knowledge. 
1

\section{ACKNOWLEDGEMENTS}

This study was supported by research funds from the European Research Council (FP7/2007-2013 Grant Agreement no. 339152). 


\section{REFERENCES}

Alcorn, S. (1932). The Tadoma method. Volta Review, 34:195-198

Arnal, L.H., Morillon, B., Kell, C.A. \& Giraud, A.L. (2009). Dual neural routing of visual facilitation in speech processing. The Journal of Neuroscience, 29(43):13445-13453

Aruffo, C., \& Shore, D.I. (2012). Can you McGurk yourself? Self-face and self-voice in audiovisual speech. Psychonomic Bulletin \& Review, 19:66-72

Baart, M., Stekelenburg, J. J. \& Vroomen, J. (2014). Electrophysiological evidence for speech-specific audiovisual integration. Neuropsychologia, 65:115-211

Baart, M., \& Samuel, A. G. (2015). Turning a blind eye to the lexicon: ERPs show no cross-talk between lip-read and lexical context during speech sound processing. Journal of Memory and Language, $85: 42-59$

Baart, M. (2016). Quantifying lip-read induced supression and facilitation of the auditory N1 and P2 reveals peak enhancements and delays. Psychophysiology, 53(9):1295-306

Benoît, C., Mohamadi, T. \& Kandel, S. (1994). Effects of phonetic context on audio-visual intelligibility of French speech in noise. Journal Speech Hearing Research, 37:1195-1203

Besle, J., Fort, A., Delpuech, C. \& Giard, M.H. (2004). Bimodal speech: early suppressive visual effects in human auditory cortex. European journal of Neuroscience, 20:2225-2234

Boersma, P. \& Weenink, D. (2013). Praat: doing phonetics by computer. Computer program, Version 5.3.42, retrieved 2 March 2013 from 〈http://www.praat.org/〉

Burfin, S., Pascalis, O., Ruiz Tada, E., Costa, A., Savariaux, C. \& Kandel S. (2014). Bilingualism affects the audio-visual processing of non-native phonemes. Frontiers in Psychology (Research Topic "New advances on the perception and production of non-native speech sounds" - Section Language Sciences), 5: 1179

Callan, D.E., Jones, J.A., Munhall, K.G., Callan, A.M., Kroos, C. \& Vatikiotis-Bateson, E. (2003). Neural processes underlying perceptual enhancement by visual speech gestures. Neuro Report, 14:22132217

Callan, D.E., Jones, J.A., Munhall, K.G., Callan, A.M., Kroos, C. \& Vatikiotis-Bateson, E. (2004). Multisensory integration sites identified by perception of spatial wavelet filtered visual speech gesture information. Journal of Cognitive Neuroscience, 16:805-816

Calvert, G.A. \& Campbell, R. (2003). Reading speech from still and moving faces: the neural substrates of visible speech. Journal of Cognitive Neuroscience, 15:57-70

Calvert, G.A., Campbell, R. \& Brammer, M.J. (2000). Evidence from functional magnetic resonance imaging of crossmodal binding in the human heteromodal cortex. Current Biology, 10(11):649-657 
Campbell, R., MacSweeney, M., Surguladze, S., Calvert, G., McGuire, P., Suckling, J., Brammer, M.J. \& David, A.S. (2001). Cortical substrates for the perception of face actions: an fMRI study of the specificity of activation for seen speech and for meaningless lower-face acts (gurning).Cognitive Brain Research, 12:233-243

Fowler, C. \& Dekle, D. (1991). Listening with eye and hand: crossmodal contributions to speech perception. Journal of Experimental Psychology- Human Perception and Performance, 17:816-828

Frtusova, J. B., Winneke, A. H., \& Phillips, N. A. (2013). ERP evidence that auditory-visual speech facilitates working memory in younger and older adults. Psychology and Aging, 28(2), 481-494

Gick, B., Jóhannsdóttir, K.M., Gibraiel, D. \& Mühlbauer, M. (2008). Tactile enhancement of auditory and visual speech perception in untrained perceivers. Journal of Acoustical Society of America, $123: 72-76$

Hickok, G. \& Poeppel, D. (2007). The cortical organization of speech processing. Nature Review Neurosciences, 8:393-402

Hommel, B., Musseler, J., Aschersleben, G. \& Prinz, W. (2001). The theory of event oding (TEC): A framework for perception and action planning. Behavioral and Brain Sciences, 24:849-878

Jones, J.A. \& Callan, D.E (2003). Brain activity during audiovisual speech perception: an fMRI study of the McGurk effect. Neuro report, 14:1129-1133

Kaganovich, N., \& Schumaker, J. (2014). Audiovisual integration for speech during mid-childhood: Electrophysiological evidence. Brain and Language, 139:36-48

Klucharev, V., Möttönen, R. \& Sams, M. (2003). Electrophysiological indicators of phonetic and nonphonetic multisensory interactions during audiovisual speech perception. Brain Res. Cogn. Brain Res., 18:65-75

Liberman, A.M. \& Mattingly, I.G (1985). The motor theory of speech perception revised. Cognition, 21:1-36

McGurk, H. \& MacDonald, J. (1976). Hearing lips and seeing voices. Nature, 264:746-748

Näätänen, R. \& Picton, T.W. (1987). The N1 wave of the human electric and magnetic response to sound: a review and an analysis of the component structure. Psychophysiology, 24:375-425

Ojanen, V., Möttönen, R., Pekkola, J., Jääskeläinen, I.P., Joensuu, R., Autti, T. \& Sams, M. (2005). Processing of audiovisual speech in Broca'sarea.Neurolmage, 25:333-338

Pekkola, J., Laasonen, M., Ojanen, V., Autti, T., Jääskeläinen, L.P., Kujala, T. \& Sams, M. (2006). Perception of matching and conflicting audiovisual speech in dyslexic and fluent readers: an fMRI study at 3T. Neurolmage, 29(3):797-807

Pickering, M.J. \& Garrod, S. (2013). An integrated theory of language production and comprehension. Behav. Brain Sci., 36:329-347 
Pilling, M. (2010). Auditory event-related potentials (ERPs) in audiovisual speech perception. Journal of Speech, Language, and Hearing Research, 52(4):1073-1081

Prinz, W. (1997). Perception and action planning. European Journal of Cognitive Psychology, 9:129154

Reed, C.M., Rabinowitz, W.M., Durlach, N.I., Braida, L.D., Conway-Fithian, S. \& Schultz, M.C. (1985). Research on the Tadoma method of speech communication. J Acoust Soc. Am., 77(1):247-257

Reed, C.-M., Rabinowitz, W.-M., Durlach, N.-I., Delhorne, L.-A., Braida, L.-D., Pemberton, J.-C., Mulcahey, B.-D. \& Washington, D.-L. (1992). Analytic study of the Tadoma method: Improving performance through the use of supplementary tactual displays. Journal of Speech and hearing Research, 35:450-465

Reisberg, D., McLean, J. \& Goldfield, A. (1987). Easy to hear but hard to understand: A lip-reading advantage with intact auditory stimuli. In B. Dodd \& R. Campbell (Eds.), Hearing by Eye: The Psychology of LipReading, 97-114

Sato, M., Buccino, G., Gentilucci, M. \& Cattaneo, L. (2010). On the tip of the tongue: modulation of the primary motor cortex during audiovisual speech perception. Speech Communication, 52(6): 533-541

Scherg, M., and VonCramon, D. (1986). Evoked dipole source potentials of the human auditory cortex. Electroencephalogr. Clin. Neurol., 65:344-360

Schwartz, J.L. \& Savariaux, C. (2001). Is it Easier to Lipread One's Own Speech Gestures Than Those of Somebody Else? It Seems Not! Auditory-Visual Speech Processing, Aalborg, Denmark, 18-23

Schwartz, J.L., Ménard, L., Basirat, A. \& Sato, M. (2012). The Perception for Action Control Theory (PACT): a perceptuo-motor theory of speech perception. Journal of Neurolinguistics, 25(5):336-354

Skipper, J., Van Wassenhove, V, Nussman, H. \& Small, S. (2007). Hearing lips and seeing voices: How cortical areas supporting speech production meditate audiovisual speech perception. Cerebral Cortex, 17:2387-2399

Skipper, J.I., Nusbaum, H.C. \& Small, S.L. (2005). Listening to talking faces: motor cortical activation during speech perception. Neurolmage, 25:76-89

Stekelenburg, J.J. \& Vroomen, J. (2007). Neural correlates of multisensory integration of ecologically valid audiovisual events. Journal of Cognitive Neuroscience, 19:1964-1973

Sumby, W.H. \& Pollack, I. (1954). Visual contribution to speech intelligibility in noise. The Journal of the Acoustical Society of America, 26:212-215

Treille, A., Cordeboeuf, C., Vilain, C. \& Sato, M. (2014a). Haptic and visual information speed up the neural processing of auditory speech in live dyadic interactions. Neuropsychologia, 57:71-77

Treille, A., Vilain, C. \& Sato, M. (2014b). The sound of your lips: electrophysiological cross-modal interactions during hand-to-face and face-to-face speech perception. Frontiers in Psychology, $5(420): 1-9$ 
Tye-Murray, N., Spehar, B., Myerson, J., Hale, S. \& Sommers, M.S. (2013). Reading your own lips: Common coding theory and visual speech perception. Psychonomic Bulletin \& Review, 20:115-119 Tye-Murray, N., Hale, S., Spehar, B., Myerson, J., \& Sommers, M. (2014). Lipreading in school-age children: The roles of age, hearing status, and cognitive ability. Journal of Speech, Language, and Hearing Research, 57:556-565

van Wassenhove, V., Grant, K.W. \& Poeppel, D. (2005). Visual speech speeds up the neural processing of auditory speech. Proceedings of the National Academy of Sciences U.S.A., 102:1181-1186

Vroomen, J. \& Stekelenburg, J.J. (2010). Visual anticipatory information modulates multisensory interactions of artificial audiovisual stimuli. Journal of Cognitive Neuroscience, 22:1583-1596

Watkins, K.E., Strafella, A.P. \& Paus, T. (2003). Seeing and hearing speech excites the motor system involved in speech production. Neuropsychologia, 41:989-994

Watkins, K.E. \& Paus, T. (2004). Modulation of motor excitability during speech perception: the role of Broca's area. Journal of Cognitive Neuroscience, 16(6):978-987 
Click here to access/download Supplementary Material s05-pa-2-AV_norm.avi 
Click here to access/download Supplementary Material s07-ta-2-AV_norm.avi 
Click here to access/download Supplementary Material s12-ka-3-AV_norm.avi 